\title{
DIAGNOSTIC TEST OF POLYNEUROPATHY SCORE, 10-G SEMMES WENSTEIN MONOFILAMENT (SWM) AND VIBRATION PERCEPTION TRESHOLD (VPT)- QUANTITATIVE SENSORY TESTING (QST) IN DISTAL SENSORY POLYNEUROPATHY (DSP)-HUMAN IMMUNODEFICIENCY VIRUS (HIV)
}

\author{
Bethasiwi Purbasari ${ }^{1}$, Shahdevi Nandar Kurniawan ${ }^{1}$, Machlusil Husna ${ }^{1}$, Didi Candradikusuma ${ }^{2}$, Harun Al-Rasyid ${ }^{3}$
}

Correspondence: bethasiwi@gmail.com

${ }^{I}$ Department of Neurology Faculty of Medicine Brawijaya University, Malang, Indonesia. ${ }^{2}$ Department of Internal Medicine Faculty of Medicine Brawijaya University, Malang, Indonesia.

${ }^{3}$ Department of Public Health Faculty of Medicine Brawijaya University, Malang, Indonesia.

Article History:

Received: September 22, 2017

Accepted: September 21, 2018

Published: January 1, 2019

\section{Cite this as:}

Purbasari B, Kurniawan SN,

Husna M, Candradikusuma D,

Al-Rasyid H. Diagnostic test of polyneuropathy score, $10-\mathrm{g}$

semmes wenstein monofilament (swm) and vibration perception treshold (vpt)-quantitative sensory testing (qst) in distal sensory polyneuropathy (dsp)human immunodeficiency virus (hiv). Malang Neurology Journal; 2019.5:21-29. DOI:

http://dx.doi.org/10.21776/ub.mnj .2019.005.01.4

\section{ABSTRACT}

Background: HIV/AIDS is a worldwide pandemic. Peripheral neuropathy is the most common neurologic complication with distal sensory polyneuropathy (HIV-DSP) as the most frequent form of manifestation. HIV-DSP is one of the major causes of global pain morbidity and often undiagnosed earlier, resulting in treatment given when HIV-DSP grade is already severe. Early detection is imperative to prevent the progression of HIV-DSP and improve patient's quality of life. Polyneuropathy score, 10-g Semmes-Weinstein Monofilament (SWM) and vibration perception threshold (VPT)-quantitative sensory testing (QST) are considered to be potential candidates for HIVDSP screening tool.

Objective: To measure the reability of Polyneuropathy Score, SWM and VPT- QST in diagnosing HIV-DSP.

Methods: This research is a descriptive study with cross-sectional design. Research was conducted on 77 patients of HIV/AIDS outpatient clinic in RS Saiful Anwar (RSSA) during November 2016 - April $2017(\mathrm{n}=77)$. Patients were screened for HIV-DSP by using polyneuropathy score, such as: Michigan Neuropathy Screening Instrument (MNSI), Toronto Clinical Scoring Sysytem (TCSS), Brief Peripheral Neuropathy Score (BPNS), SWM, and VPT-QST. Sensitivity, specificity, positive predictive value and negative predictive value of BPNS, TCSS, MNSI, SWM and VPT-QST are analyzed using cross tabulation. Area under receiver operating characteristic (ROC) curve analysis was used to measure diagnostic accuracy of each tools. SPSS 19.0 was used for statistical analysis.

Results: TCSS had a sensitivity, specificity, positive predictive value, negative predictive value positive likelihood ratio, negative likelihood ratio, and accuracy as much as: $70 \%, 97 \%, 97 \%, 74 \%$, $23,33,0,28$ and $83 \%$. The result of TCSS analysis using ROC showed that TCSS had excellent diagnostic value with AUC (Area Under Curve) of 0.89 (95\%CI 82\%-96,5\%). The AUC of BPNS score, monofilamen and VPT-QST respectively: 0,469, 0,194, and 0,189.

Conclusion: The result of the HIV-DSP diagnostic test using TCSS score has the best value compared to other tools.

Keywords: Distal Sensory Polyneuropathy HIV, TCSS, MNSI, BPNS, SWM, VPT-QST

\section{Introduction}

Human Immunodeficiency Virus (HIV) had become a worldwide pandemic with global prevalence of 36.9 million people living with HIV with two million new cases each year. ${ }^{1,2}$ In 2009, an estimate of 186.000 people in Indonesia is HIV positive. ${ }^{3}$ Papua, Jakarta, and Bali are Indonesia's three provinces with the highest new HIV infection cases in 2011.4

Peripheral neuropathy is one of the most commonly found neurological complication in HIV infection yet frequently remain undiagnosed, resulting in inadequate treatment. ${ }^{5,7,8}$
However, increased life expectancy from ARV therapy leads to increased prevalence of peripheral neuropathy due to longer duration of HIV infection and toxicity of ARV medications on peripheral nerves. ${ }^{6}$ From six HIVassociated peripheral neuropathy clinical manifestation, the most prevalent neurologic complication is distal sensory polyneuropathy (HIV-DSP).

Prevalence rate of HIV-DSP varied worldwide with estimated range of $27 \%-57 \%$, where $38 \%-90 \%$ HIV-DSP patients experienced neuropathic pain. ${ }^{11,12}$ HIV-DSP neuropathic pain is frequently debilitating and adversely impacting patients' quality of life, ${ }^{13}$ also relatively difficult 
to treat. ${ }^{14}$ As a result, HIV-DSP become one of the major causes of global pain morbidity. Early recognition of signs and symptoms of HIV-DSP is imperative to prevent the progression and to improve patients' quality of life. Unfortunately, HIV-DSP frequently undiagnosed in early stages, resulting in treatment provided when HIV-DSP grade is already severe. ${ }^{6}$

Until today, there had been no gold standard diagnostic testing for establishing HIV-DSP diagnosis. ${ }^{15}$ Nerve conduction velocity (NCV) test using electroneuromyography (ENMG), ${ }^{16-17}$ quantitative sensory test (QST), ${ }^{18-19}$ and intra epidermal nerve fiber (IENF) density assessment ${ }^{20}$ had been heavily investigated and proven to assist in HIV-DSP diagnosis. The aforementioned tests are not feasibly conducted for early screening HIVDSP to all HIV patients due to the high cost and relatively lengthy period of test. Thus, the need for a simple, easy, effective, and efficient test to diagnose HIV-DSP in its early stage.

Several diagnostic tests had been proposed as distal sensory polyneuropathy early screening instrument in various cases worldwide, such as Polyneuropathy Scoring using Brief Peripheral Neuropathy Screen (BPNS), ${ }^{21-23}$ Toronto Clinical Scoring System $^{25}$ (TCSS), and Michigan Neuropathy Screening Instrument (MNSI), ${ }^{24,26-28}$ also monofilament examination using 10-g Semmes-Weinstein Monofilament $(\mathrm{SWM}){ }^{29}$ Meanwhile, DSP diagnostic modality using easily-used diagnostic instrument such as portable quantitative sensory testing (QST) had been validated in numerous research studies. ${ }^{30}$ However, there had been no research comparing the diagnostic reliability of these screening instruments to EMNG in detecting HIVDSP.

The objective of this research is to determine the reliability of HIV-associated distal sensory neuropathy (HIV-DSP) diagnostic screening instruments, SWM, and VPT-QST compared to NCV testing using ENMG in RSU Saiful Anwar (RSSA) Malang.

\section{Methods}

\section{Design}

This research is designed as an observational research using cross sectional method.

\section{Location and Timing}

This research was conducted in tropical infection clinic Saiful Anwar General Hospital Malang from November 2016 to June 2017 upon receiving approval from Saiful Anwar General Hospital Malang Ethical Committee.

\section{Population and Sample}

The participant of this research are patients meeting inclusion and exclusion criteria, using purposive sampling. Inclusion criteria for this research are patients with confirmed HIV diagnosis based on the following 3 (three) methods, Reagen SD Bioline HIV 1/ 2 3.0, Intec One Step Anti-HIV (1\&2), and Oncoprobe, patients with confirmed DSP diagnosis based on clinical examination and ENMG, aged between 18-64 years old, and gave informed consent to participate in polyneuropathy scoring examination, SWM, VPT-QST and ENMG. Exclusion criteria for this research are patients with lower limbs defect, pregnant, suffering from chronic illness, suffering from cardiovascular disease (hypertension, coronary disease, and history of stroke), history of cancer and/or chemoteraphy, history of alcohol consumption, history of tuberculosis medication in the last 6 (six) months, history of diabetes, contraindication to electrodiagnostic examination, neurological deficit, absence of peripheral artery disease indicated by pulsation detection of a.dorsalis pedis, and patients not giving informed consent to participate in polyneuropathy scoring examination, SWM, VPT-QST and ENMG.

\section{Variable Definition}

\section{Brief Peripheral Neuropathy Screen (BPNS)}

A DSP scoring tool consisted of 3 (three) main components: symptoms experienced by patients, vibration perception test using tuning fork, and achilles tendon reflex testing. BPNS score $0=$ no DSP (normal), BPNS score 1$3=$ mild DSP (grade 1), BPNS score 4-6=moderate DSP (grade 2), BPNS score 7-10=severe DSP (grade 3).

\section{Toronto Clinical Scoring System (TCSS)}

A DSP scoring tool consisted of 3 (three) components, symptom scores, reflex scores, and sensory test scores. Score $\leq 5$ no neuropathy, score $6-8=$ mild neuropathy, score $9-11=$ moderate neuropathy and score $\geq 12$ indicated severe neuropathy.

\section{Michigan Neuropathy Screening Instrument (MNSI)}

A DSP scoring tool consisted of 2 (two) components, symptoms experienced by patients and physical examination by medical professional. MNSI score $\geq 9.5$ (7 on questionnaire and 2.5 on physical examination) indicated DSP.

\section{0-g Semmes-Weinstein Monofilament (SWM)}

A cutaneuos sensation evaluation using 5,07 gauge Semmes Weinstein monofilament fiber (with 10 grams of force to buckle) on 9 spots at plantar pedis and 1 spot at dorsum pedis. Abnormal result defined by failure to detect pressure in $\geq 4$ sites.

\section{Vibration perception treshold (VPT) - quantitative sensory testing (QST)}

A quantitative sensory testing to determine patient's ability to detect vibration perception treshold with predefined intensity. The vibratory transducer was placed on the dorsal surface of the great toe. The output is categorized according to pre-programmed degree of abnormality, adjusted to patient's age. Normal range $=8-12$, abnormal range $=0-7$.

\section{Confirmed HIV-DSP}

Presence of polyneuropathy signs or symptoms started in distal body parts, symmetrically appeared, and abnormality in ENMG test.

HIV-DSP degree defined as follows: a. Mild: pinprick diminished at the tip of the toes or vibration detected for 510 seconds at the great toe. Moderate: pinprick diminished to the ankles or vibration detected for $<5$ seconds at the great toe. Severe: pinprick diminished past the ankles or no vibration detected at the great toe. Electroneuromyography test performed by testing the sural, median, and ulnar sensory nerves and testing the tibialis posterior, peroneal, median, and ulnar motor nerves. Test performed by neurophysiology professional using Nihon Kohden/Neuropack M1 MEB-9200 version 08.06 (copyright 1997-2007) at electrophysiology laboratory 
RSSA Malang. To prevent bias, participants' identity is restricted during NCV test.

\section{Procedure and Workflow}

At the beginning stage of research, patients completed a form containing required data for this research. Data required including patients' identity, short anamnesis to obtain information on risk factors, duration of HIV, duration of ARV teraphy, types of medication consumed, associated comorbidities (hypertension, history of stroke, coronary disease, metabolic syndrome), other neuropathy risk factors (malnutrition, vitamin B12 deficiency, history of cancer and/or chemoteraphy, history of alcohol consumption, history of neurotoxic medications, and history of peripheral nerve entrapment. Afterwards, vital sign examination (blood pressure, pulse, and respiratory rate), internal status examination, and neurological status examination are performed. Subsquently, an examination to eliminate peripheral neuropathy due to nerve entrapment by performing provocative maneuvers, consisted of Lasegue, Bragard, Sicard, Valsalva, Lhermitte, and Spurling.

The next step is to determine whether patients meeting the inclusion criteria. Patients meeting the inclusion criteria further eliminated using exclusion criteria. The remaining patients that are willing to participate asked to fill out informed consent form. Afterwards. HIV-DSP signs and symptoms clinical examination is performed. Upon encountering abnormality, further procedure of neuropathy scoring consisted of BPNS, TCSS, and MNSI is performed. Upon completion, subsequent procedure of 10-g SemmesWeinstein Monofilament (SWM) using neuropen (Owen Mumford, LTD) at plantar pedis and dorsum pedis and vibration perception treshold (VPT) - quantitative sensory testing (QST) using Nervecheck (Phi Med Europe S.L. Barcelona, Spain) at distal lower extremity. Upon completion of preceding DSP tests, nerve conduction velocity test to confirm HIV-DSP diagnosis is performed using electroneuromyography (Nihon Kohden/Neuropack M1 MEB-9200 version 08.06). All tests performed by trained medical professional and the result of all test are recorded and documented for further analysis.

\section{Data Analysis}

Data obtained in this research is statistically analyzed using SPSS 19.0. Data analysis required are sensitivity, specificity, positive predictive value and negative predictive value of BPNS, TCSS, MNSI, SWM and VPT-QST compared to NCV-ENMG using cross tabulation $(2 \times 2)$. Under the curve receiver operating characteristic (ROC) area analysis also performed to compare diagnostic accuracy of each HIV-DSP diagnostic tool.

\section{Results}

\section{Subject Characteristic}

This research involved 77 patients meeting inclusion criteria, not meeting exclusion criteria, and able to participate throughout the whole research duration as research subject. HIV-DSP prevalence were detected in $53 \%$ of the patients, with $21 \%$ mild neuropathy, $21 \%$ moderate neuropathy and $11 \%$ severe neuropathy. Percentage of HIV patients with no HIV-DSP based on predetermined diagnosis criteria is $47 \%$.

Basic profile of polyneuropathy HIV-DSP patients in this research is shown in Table 1 below. Average patients age is 35.08 years old for normal group and 39.22 years old for HIV-DSP group. Average time since HIV diagnosis is 1.92 years for normal group and 2.20 years for HIV-DSP group. Average duration of ARV teraphy is 1.60 years for normal group and 1.82 years for HIV-DSP group. Average CD4 nadir count is 443 cells $/ \mathrm{mm}^{3}$ for normal group and 141,46 cells $/ \mathrm{mm}^{3}$ for HIV-DSP group. Average current CD4 count is 463,83 cells $/ \mathrm{mm}^{3}$ for normal group and 228,95 cells $/ \mathrm{mm}^{3}$ for HIV-DSP group. Male-female proportion between two groups are similar with 18:18 for normal group and 17:24 for group with HIV-DSP. Smoker and non-smoker patient proportion varies between the two groups, with 2:34 for normal group and 12:29 for group with HIV-DSP.

Table 1. Basic Profile of HIV-DSP Patients Analysis Result.

\begin{tabular}{crrrrr}
\hline Basic Data & Normal $(\mathbf{n}=\mathbf{3 6})$ & \multicolumn{2}{c}{ HIV- DSP $(\mathbf{n}=\mathbf{4 1})$} \\
Age (years) & 35.08 & \pm 9.07 & 39.22 & \pm 10.79 \\
HIV duration (years) & 1.92 & \pm 2.18 & 2.20 & \pm 1.85 \\
ARV therapy duration (years) & 1.60 & \pm 2.14 & 1.82 & \pm 1.80 \\
Body Mass Index & 20.90 & \pm 3.49 & 21.85 & \pm 4.23 \\
CD4 Nadir (average \pm stdev) & 443.00 & \pm 508.73 & 141.46 & \pm 156.63 \\
CD4 (average \pm stdev) & 463.83 & \pm 123.62 & 228.95 & \pm 131.44 \\
Sex (female, male) & $18: 18$ & $17: 24$ \\
Smoking (positive,negative) & $2: 34$ & $12: 29$ \\
\hline
\end{tabular}

\section{HIV-DSP Result Using Polyneuropathy Diagnostic Scoring, Monofilament and VPT-QST Test}

HIV-DSP prevalence using BPNS score, MNSI score, TCSS score, monofilament and VPT-QST test compared to actual HIV-DSP are shown in Figure 1. The highest HIVDSP prevalence is from BPNS score at $76.62 \%$ while the lowest is from MNSI score at $25.97 \%$. HIV-DSP prevalence using monofilament and VPT-QST is similar at $41.56 \%$ while TCSS score results at $38.96 \%$. 


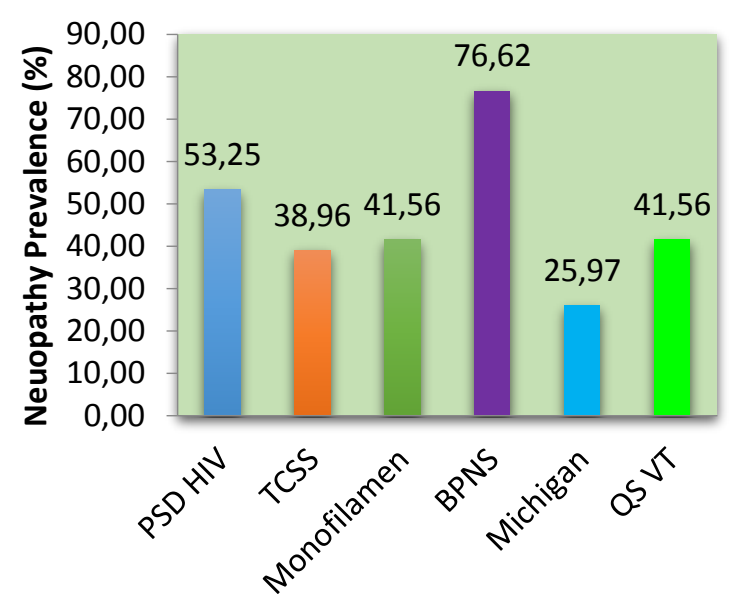

Fugure 1. HIV-DSP Prevalence Test Result Using BPNS Score, Michigan Score (MNSI), TCSS Score, Monofilament (SWM) and VPT-QST Test.

Cross tabulation of BPNS score, MNSI score, TCSS score, Monofilament and VPT-QST Examination are shown in Table 2. Based on the table, sensitivity value for BPNS is $100 \%$, with $50 \%$ specificity, $69 \%$ positive predictive value, $100 \%$ negative predictive value, positive likelihood ratio of 2.0, negative likelihood ratio of 0 , and $77 \%$ accuracy. Meanwhile sensitivity value for MNSI is $46 \%$, with $97 \%$ specificity, $95 \%$ positive predictive value, $61 \%$ negative predictive value, positive likelihood ratio of 15.3 , negative likelihood ratio of 0.03 , and $70 \%$ accuracy. TCSS sensitivity value is $70 \%$, with $97 \%$ specificity, $97 \%$ positive predictive value, $74 \%$ negative predictive value, positive likelihood ratio of 23.33, negative likelihood ratio of 0.28 , and $83 \%$ accuracy. As for monofilament, sensitivity value is $66 \%$, with $86 \%$ specificity, $84 \%$ positive predictive value, $69 \%$ negative predictive value, positive likelihood

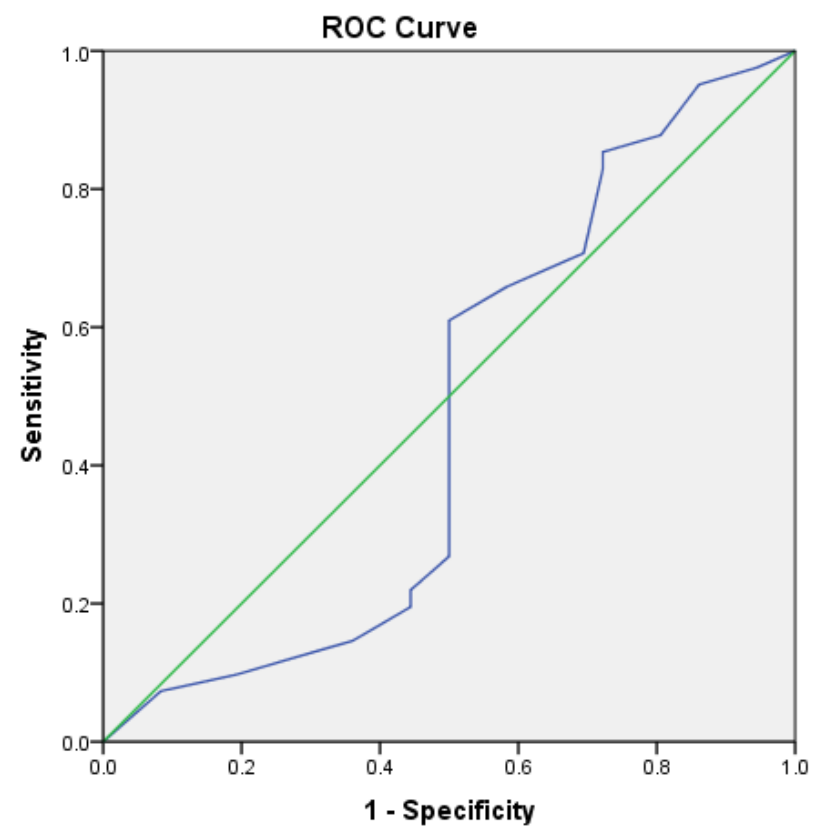

$\mathbf{A}$ ratio of 7.1 , negative likelihood ratio of 0.39 , and $77 \%$ accuracy. Lastly, sensitivity value for VPT-QST is $73 \%$, with $94 \%$ specificity, $94 \%$ positive predictive value, $76 \%$ negative predictive value, positive likelihood ratio of 12.16 , negative likelihood ratio of 0.29 , and $83 \%$ accuracy.

Table 2. Cross Tabulation of BPNS Score, MNSI Score, TCSS Score, Monofilament and VPT-QST Test.

\begin{tabular}{|c|c|c|c|}
\hline & HIV-DSP & Non HIV-DSP & \\
\hline \multicolumn{4}{|l|}{ BPNS Score } \\
\hline \multirow{2}{*}{$\begin{array}{l}\text { HIV-DSP } \\
\text { Non HIV-DSP }\end{array}$} & 41 & 18 & 59 \\
\hline & 0 & 18 & 18 \\
\hline \multicolumn{4}{|l|}{ MNSI Score } \\
\hline \multirow{2}{*}{$\begin{array}{l}\text { HIV-DSP } \\
\text { Non HIV-DSP }\end{array}$} & 19 & 1 & 20 \\
\hline & 22 & 35 & 57 \\
\hline \multicolumn{4}{|l|}{ TCSS score } \\
\hline \multirow{2}{*}{$\begin{array}{l}\text { HIV-DSP } \\
\text { Non HIV-DSP }\end{array}$} & 29 & 1 & 30 \\
\hline & 12 & 35 & 47 \\
\hline \multicolumn{4}{|l|}{ SWM } \\
\hline HIV-DSP & 27 & 5 & 32 \\
\hline Non HIV-DSP & 14 & 31 & 45 \\
\hline \multicolumn{4}{|l|}{ VPT-QST } \\
\hline HIV-DSP & 30 & 2 & 32 \\
\hline Non HIV-DSP & 11 & 34 & 45 \\
\hline
\end{tabular}

Diagnostic test result of BPNS Score, TCSS score, Monofilament and VPT-QST using Receiver Operating Curve (ROC) shown below in Figure 2. AUC value for BPNS is $46.9 \%$, TCSS is $89.3 \%$, Monofilament is $19.4 \%$ and VPT-QST is $18.9 \%$. The expected AUC is $60 \%$.

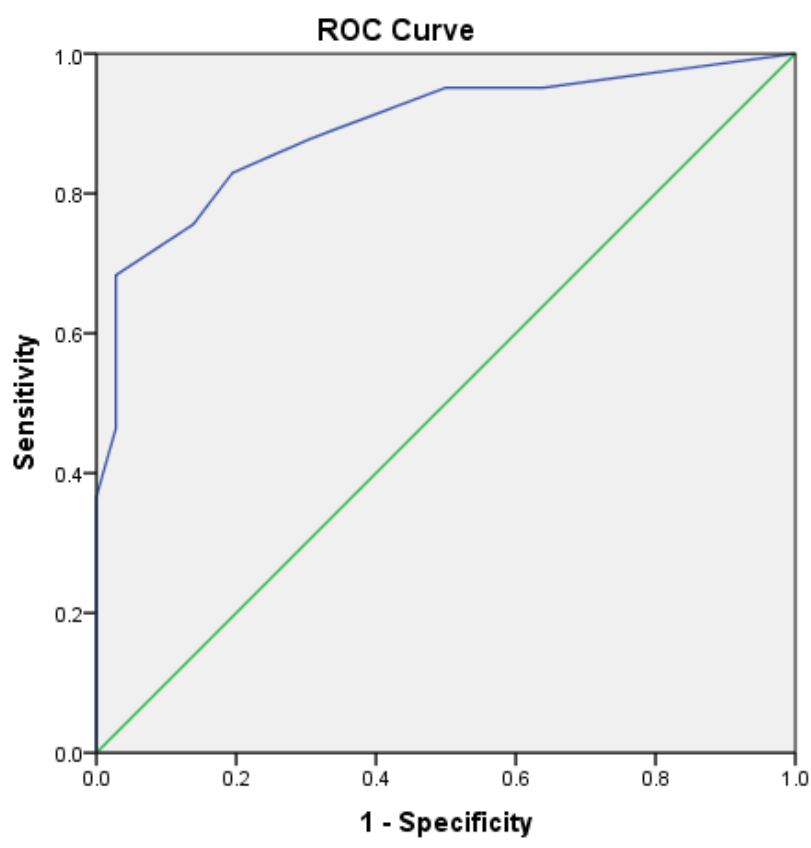

B 


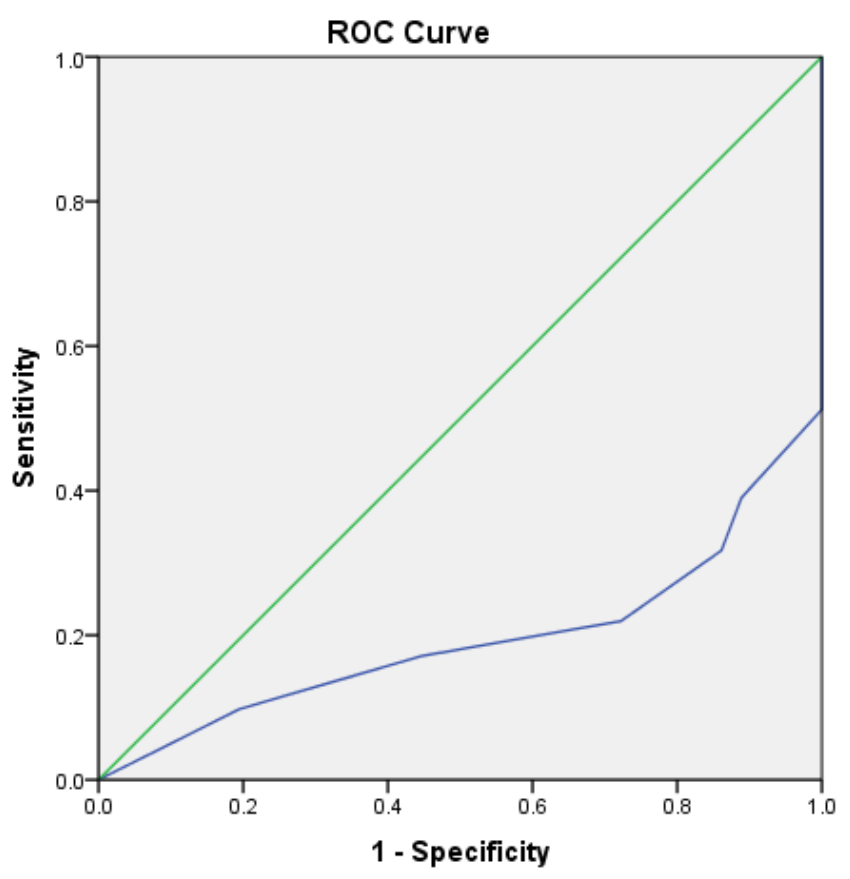

C

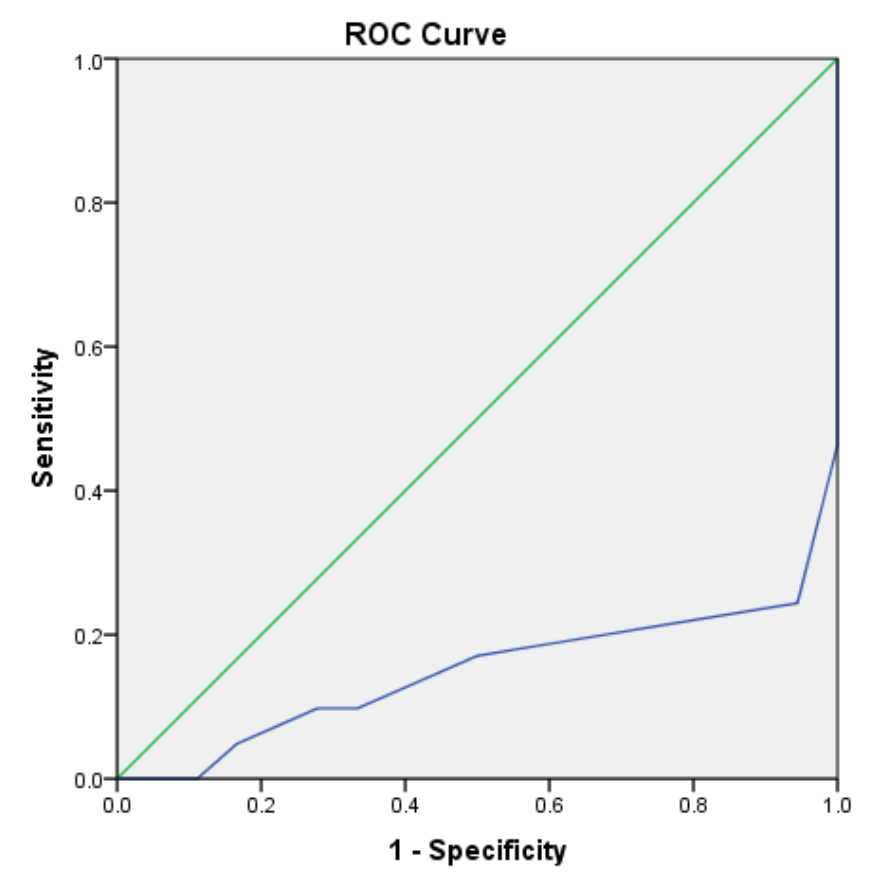

D

Figure 2. ROC Analysis Result for BPNS Score (A), TCSS Score (B), Monofilament (C) and VPT-QST Test (D).

The overall comparison of DSP-HIV diagnostic test using BPNS score, MNSI score, TCSS score, Monofilament and VPT-QST test is presented in Table 3.

Table 3. Comparison of DSP-HIV Diagnostic Test Using BPNS Score, MNSI Score, TCSS Score, Monofilament and VPT-QST

\begin{tabular}{lllllll}
\hline $\begin{array}{l}\text { Diagnostic } \\
\text { Tool }\end{array}$ & Sens & Spec & PPV & NPV & Acc & AUC \\
\hline BPNS & $100 \%$ & $50 \%$ & $69 \%$ & $100 \%$ & $77 \%$ & $46.9 \%$ \\
MNSI & $46 \%$ & $97 \%$ & $95 \%$ & $61 \%$ & $70 \%$ & - \\
TCSS & $70 \%$ & $97 \%$ & $97 \%$ & $74 \%$ & $83 \%$ & $89.3 \%$ \\
SWM & $66 \%$ & $86 \%$ & $84 \%$ & $69 \%$ & $75 \%$ & $19.4 \%$ \\
VPT-QST & $73 \%$ & $94 \%$ & $94 \%$ & $76 \%$ & $83 \%$ & $18.9 \%$
\end{tabular}

Abbreviations: Sens: Sensitivity; Spec: Specificity; PPV: Positive Predictive Value; NPV: Negative Predictive Value; Acc: Accuracy; AUC: Area Under the Curve

\section{Discussion}

This research is conducted by performing HIV-DSP screening on HIV patients treated in infection clinic RSU Saiful Anwar Malang, with total sample of 77 patients. Based on PNP criteria from AAN, AAEM and AAPM\&R, HIV-DSP prevalence were detected in $53 \%$ of the patients, with $21 \%$ mild neuropathy, $21 \%$ moderate neuropathy and $11 \%$ severe neuropathy. Average patient age is 35.08 years old for normal group and 39.22 years old for HIV-DSP group, with no significant age difference between the two groups. The average age of patients with HIV-DSP in this research is similar with other research where the average age of patients with HIV-DSP is 31.81 years old for group without neuropathy and 34.11 years old for group with neuropathy. ${ }^{31}$ Male-female proportion between the two groups is similar with 18:18 for normal group and 17:24 for group with HIV-DSP, it is generally consistent with other research, where there were no significant difference in sex percentage of HIV-DSP patients. ${ }^{14,32}$ Average years since HIV diagnosis is 1.92 years for normal group and 2.20 years for HIV-DSP group. Research conducted by Konchalard, et. al. shown average years since HIV diagnosis is 3.2 years for normal group and 3.7 years for group with HIV neuropathy, with no significant difference between the two group. ${ }^{33}$ Body mass index for both group is relatively similar, with 20.9 for normal group and 21.85 for HIV-DSP group, consistent with other research where there is no significant BMI difference between neuropathy group dan non-neuropathy HIV group. ${ }^{14}$

Average CD4 nadir count is 443 cells $/ \mathrm{mm} 3$ for normal group and 141.46 cells/mm3 for HIV-DSP group. The result is relatively consistent with other research, where CD4 nadir count is 256 cells/mm3 for group without HIV neuropathy and 96 cells/mm3 for group with HIV neuropathy, indicating significant CD4 nadir count difference between two groups. ${ }^{33}$ Average current CD4 count is 463.83 cells $/ \mathrm{mm}^{3}$ for normal group and 228.95 cells $/ \mathrm{mm}^{3}$ for HIV-DSP group. This result is also consistent with other research, where significant current CD4 count difference between group without HIV neuropathy and group with HIV neuropathy is observed. ${ }^{34}$

This research screened HIV-DSP using BPNS score, MNSI score, TCSS score, monofilament test using 10-g Semmes Weinstein, and vibration perception using VPT-QST. HIVDSP prevalence comparison in this research using BPNS score, MNSI score, TCSS score, monofilament test, and VPT-QST test with gold standard of diagnosis criteria and ENMG were as follows 76.62\%, 25.97\%, 38.96\%, 41.56\%, $41.56 \%$ and $53.25 \%$. HIV-DSP prevalence using BPNS score at $76.62 \%$ is relatively high compared to gold standard. This result was possibly due to the high sensitivity of this tool, thus patients with minimum clinical 
condition is classified in HIV-DSP group while the clinical signs and ENMG test shown normal result.

This research shown the following values for BPNS score: sensitivity is $100 \%, 50 \%$ specificity, $69 \%$ positive predictive value, $100 \%$ negative predictive value, positive likelihood ratio of 2.0, negative likelihood ratio of 0 , and $77 \%$ accuracy. BPNS diagnostic result using Receiver Operating Curve (ROC) shown Area Under Curve (AUC) value of 0.469 (95\% IK $33.22 \%-60.6 \%$ ), sig value 0.639 . AUC value of $46.9 \%$ translated to where BPNS is used to diagnose neuropathy in $100 \mathrm{HIV}$ patients, 47 patients will have confirmed diagnosis. Based on its confidence interval, AUC value for BPNS in population ranged from $33.22 \%$ to $60.6 \%$. From statistical perspective, the value is considered as unsatisfactory. The result is relatively different from other research where BPNS diagnostic values for HIV-DSP were as follows: Sensitivity is $17-18 \%, 93-94 \%$ specificity, $10-37 \%$ positive predictive value, $84-96 \%$ negative predictive value, positive likelihood ratio of $2.45-2.75$, negative likelihood ratio of $0.88-0.89$, and $80-89 \%$ accuracy. Meanwhile BPNS AUC from the research were at $0.553-0.554 .^{35}$ The different diagnostic test result were possibly due to substantial difference in sample size. This research sample size is 77 patients while research by Cettomai, et. al., recorded sample size of 240 patients. The significant sample size difference considered to be the cause of BPNS diagnostic values for HIV-DSP result difference.

For MNSI score, the values for HIV-DSP diagnostic test are as follows: sensitivity is $46 \%$, $97 \%$ specificity, $95 \%$ positive predictive value, $61 \%$ negative predictive value, positive likelihood ratio of 15.3 , negative likelihood ratio of 0.03 , and $70 \%$ accuracy. As of today, MNSI score had never been utilized to diagnose HIV-DSP, and only used as screening method for patients with polyneuropathy diabetes mellitus (PNP-DM). Several research reported MNSI score sensitivity value at $61 \%, 79 \%$ specificity, $55 \%$ positive predictive value, and negative predictive value at $83 \% .^{36}$ The difference of diagnostic value were possibly due to different sample size and different distal sensory neuropathy examined.

The values for HIV-DSP diagnostic test for monofilament test are as follows: sensitivity is $66 \%, 86 \%$ specificity, $84 \%$ positive predictive value, $69 \%$ negative predictive value, positive likelihood ratio of 7.1, negative likelihood ratio of 0.39 , and $77 \%$ accuracy. Monofilament test diagnostic result using Receiver Operating Curve (ROC) shown unsatisfactory diagnostic value due to less than $50 \%$ line curve value. The resulting Area Under Curve (AUC) value is 0.194 (95\% CI 9.3\%-29\%), sig value 0.000. From statistical perspective, the value is considered as unsatisfactory. Monofilament test AUC value of $19.4 \%$ translated to 19 confirmed neuropathy diagnosis in 100 HIV patients. Based on its confidence interval, Monofilament AUC value in population ranged from $9.3 \%$ to $29 \%$. Hypothesis testing performed by comparing AUC result with AUC value of $50 \%$. The resulting Sig value of $<0,05$ shown significant difference with AUC value of $50 \%$. From clinical perspective, Monofilament AUC value is unsatisfactory by not meeting the expected AUC value of $60 \%$. The result is relatively similar with other research where Monofilament HIV-DSP diagnostic values are as follows: Sensitivity at $68 \%, 93 \%$ specificity, $68 \%$ positive predictive value, 93\% negative predictive value, positive likelihood ratio of 10.18, negative likelihood ratio of 0.35 , $89 \%$ accuracy. Meanwhile, AUC value for Monofilament for the research is $0.813 .{ }^{35}$ Cross tabulation diagnostic test result shown similar result with other research but AUC value using ROC shown relatively different result. ROC shown relatively different result due to different predetermined diagnostic Monofilament cut off value. In this research, cut off is determined at $60 \%$, while research conducted by Cettomai, et. al., determined cut off at $90 \%$. The higher determined cut off value, the higher the sensitivity but the lower the specificity.

The values for HIV-DSP diagnostic test for VPT-QST test are as follows: sensitivity is $73 \%, 94 \%$ specificity, 94\% positive predictive value, $76 \%$ negative predictive value, positive likelihood ratio of 12.16, negative likelihood ratio of 0.29 , and $83 \%$ accuracy. VPT-QST diagnostic result using Receiver Operating Curve (ROC) shown unsatisfactory diagnostic value for VPT-QST due to less than 50\% line curve value. The resulting Area Under Curve (AUC) value from ROC method is 0,194 (95\%CI 5.9\%$23.9 \%$ ), sig value 0.000 . From statistical perspective, the value is considered as unsatisfactory. AUC value of $18.9 \%$ translated to if VPT-QST used to diagnose neuropathy in 100 HIV patients, 19 patients will have confirmed diagnosis. Based on its confidence interval, VPT-QST AUC value in population ranged from $5.9 \%$ to $23.9 \%$. Hypothesis testing performed by comparing AUC result with AUC value of $50 \%$. The resulting Sig value of $<0,05$ shown significant difference with AUC value of $50 \%$. From clinical perspective, VPT-QST AUC value is unsatisfactory by not meeting the expected AUC value of $60 \%$. Cross tabulation diagnostic test result shown similar result with other research, where the result for VPT-QST HIV-DSP diagnostic values are as follows: Sensitivity at 56\%, 81\% specificity, $38 \%$ positive predictive value, $90 \%$ negative predictive value, positive likelihood ratio of 2.91 , negative likelihood ratio of 0.54 , and $76 \%$ accuracy. Meanwhile, AUC value for QST-PVT for the research is $0.684 .^{35}$ Different AUC value was possibly due to significant difference in sample size and different characteristics of research sample. The sample size for this research is 77 patients, while the sample size for research conducted by Cettomai, et. al., was 238 patients. The sample size difference is considered to be a major factor in VPT-QST AUC result difference.

HIV-DSP diagnostic test result using TCSS score shown highest accuracy compared to other tools. The values for HIV-DSP diagnostic test for TCSS score are as follows: sensitivity is $77 \%, 97 \%$ specificity, $97 \%$ positive predictive value, $74 \%$ negative predictive value, positive likelihood ratio of 23.33, negative likelihood ratio of 0.28 , and $83 \%$ accuracy. TCSS score analysis result shown excellent diagnostic value due to higher than $50 \%$ line curve value. The resulting Area Under Curve (AUC) value is 0.893 (95\%CI 82\%-96.5\%), sig value 0.000. From statistical perspective, the value is considered as satisfactory. AUC value of $89.3 \%$ translated to if TCSS score utilized to diagnose neuropathy in $100 \mathrm{HIV}$ patients, 89 patients will have confirmed diagnosis. Based on its confidence interval, TCSS score AUC value in population ranged from $82 \%$ to $96.5 \%$. Hypothesis testing performed by comparing AUC result with AUC value of $50 \%$. The resulting Sig 
value of $<0,05$ shown significant difference with AUC value of $50 \%$. From clinical perspective, TCSS AUC value is satisfactory by exceeding the expected AUC value of $60 \%$. TCSS score diagnostic result is generally similar with other research where TCSS score sensitivity and specificity value for HIV-DSP is $79 \%$ with AUC value of $0,87 .^{37}$

The relatively high positive predictive value and negative predictive value from screening tools used in this research shown that positive diagnostic result majorly resulted from true positive data while negative diagnostic result majorly resulted from true negative data. In this research, the accuracy of neuropathy test score consisted of HIV-DSP signs and symptoms such as TCSS is better in diagnosing HIV-DSP compared to single modality test such as Monofilament and vibration sensation test with VPT-QST. Diagnostic test using Monofilament and vibration sensation test with VPT-QST shown similar result. This result is consistent with research conducted by Meijer et. al., where test using tuning fork resulted in similar validity and positive predictive value with Monofilament test, even produced similar result with SWM test combined with PNP-DM test score. ${ }^{38-40}$

TCSS shown the highest diagnostic value for HIV-DSP and can be reliably used as DSP screening tool on HIV patients.

A follow up research with bigger sample size and more specific sample criteria is needed to evaluate the value of other diagnostic tools.

This research's applicability is limited to HIV patients similar to this research population setting and not generalized to wider HIV patients population.

\section{Conclusion}

TCSS shown the highest diagnostic value for detecting HIV-DSP compared to other tests. The highest accuracy were shown by TCSS and VPT-QST at $83 \%$ and highest AUC value shown by TCSS at $89.3 \%$.

\section{Acknowledgement}

The author thanks to the Brawijaya University and Saiful Anwar General Hospital, Malang, East Java, Indonesia for facilitating this research.

\section{References}

1. World Health Organization. World Health Observatory (WHO) Data: HIV/AIDS. www.who.int/gho/hiv/en/. Accessed on 7 May 2016, 09.35

2. Joint United Nations Programme on HIV/AIDS. Global report 2012: Unaids report on the global AIDS epidemic. Joint United Nations Programme on HIV/AIDS, 2013

3. Kementerian Kesehatan Republik Indonesia Direktorat Jenderal Pengendalian Penyakit dan Penyehatan Lingkungan. Pedoman nasional tatalaksana klinis infeksi hiv dan terapi antiretroviral; 2011.1-2

4. Unicef Indonesia. Responding to hiv and aids. issue briefs; oktober 2012.1-3

5. Letendre SL, Ellis RJ, Everall I, Ances B, Bharti A, McCutchan. A. neurologic complications of hiv disease and their treatment. international aids societyusa: topics in hiv medicine; 2009.46-56. PubMed: https://www.ncbi.nlm.nih.gov/pubmed/19401607

6. Duarte AG, Papp JR, Simpson DM. Diagnosis and management of hiv-associated neuropathy. Neurol Clin 26, Elsevier saunders; 2008.821-832. DOI: 10.1016/j.ncl.2008.04.001

7. Kamerman PR, Wadley AL, Cherry CL. Hivassociated sensory neuropathy: risk factors and genetics. Curr Pain Headache Rep; 2012.16:226-36. DOI: $10.1007 / \mathrm{s} 11916-012-0257-\mathrm{z}$

8. Beadles WI, Jahn A, Weigel R, Clutterbuck D. Peripheral neuropathy in hiv-positive patients at an antiretroviral clinic in lilongwe, malawi. Trop Doet; 2009.39:78-80.

DOI: https://doi.org/10.1258/td.2008.080213

9. Morgello S, Estanislao L, Simpson D, Geraci A., DiRocco A., Gerita P. Manhattan hiv brain bank. hivassociated distal sensory peripheral neuropathy in the era of highly active antiretroviral theraphy. the manhattan hiv brain bank. Arch Neurol; 2004.61:153161

10. Shikuma C, Gerschenson M, Ananworanich J, Valeour V, Teeratakulpisarn N, Jadwattanakul T, et al. SEARCH 003 protocol team. Determinants of epidermal nerve fiber density in antiretroviral-naïve HIV-infected individuals. HIV Med; 2012.13:602-608

11. Ellis RJ, Rosario D, Clifford DB, McArthur JC, Simpson D, Alexander T, Gelman BB, Vaida F, Collier A., Marra CM, Ances B, Atkinson JH, Dworkin RH, Morgello S, Grant I. Continued high prevalence and adverse clinical impact of human immunodeficiency virus associated sensory neuropathy in the era of combination antiretroviral theraphy: the charter study. Arch Neurol; 2010.67: 552-58. DOI: 10.1001/archneurol.2010.76

12. Maritz J, Benatar M, Dave JA., Harrison TB, Badri M, Levitt NS, Heckmann JM. Hiv neuropathy in south african: frequency, characteristics, and risk factors. Muscle Nerve; 2010.41:599-606. DOI: 10.1002/mus.21535

13. Keltner JR, Vaida F, Ellis RJ, Moeller-Bertram T, Fitzsimmons C, Duarte NA, Robinson-Papp J, Dworkin RH, Clifford DB, Mcarthur JC, Simpson DM, Collier AC, Marra CM, Atkinson JH, Grant I. Health-related quality of life 'well-being' in hiv distal neuropathic pain is more strongly associated with depression severity than with pain intensity. Psychosomatics; 2012.53:380-86

14. Phillips TJ, Cherryl CL, Cox S, Marshall SJ, Rice ASC. Pharmacological treatment of painful hivassociated sensory neuropathy: a systematic review and metaanalysis of randomized controlled trials. PloS One; 2010.5:e14433. DOI: 10.1371/journal.pone.0014433

15. Brew BJ. The peripheral nerve complication of human immunodeficiency virus (hiv) infection. Muscle Nerve; 2003.28:542-552

16. Malessa R, Agelink M, Himmelmann M, Kloss T, Mertins L, Brockmeyer N. Nerve conduction changes in asymptomatic hiv-1 seropositive individuals in the absence of other risk factors for neuropathy. Electromyogr Clin Neurophysiol; 1996.26:3-8. PubMed: 
https://www.ncbi.nlm.nih.gov/pubmed/8654319

17. Barohn R, Gronseth G, Amato A., et al. Cerebrospinal fluid and nerve conduction abnormalities in hiv positive individuals. J Neurol Sci; 1996.136:81-5

18. Phillips TJC, Brown M, Ramirez JD, Perkins J, Woldeamanuel YW, Williams AC.de C, et al. Sensory, psychological, and metabolic dysfunction in hiv-associated peripheral neuropathy: a cross sectional deep profiling study. PAIN; 2014.155:1846-60. DOI: 10.1016/j.pain.2014.06.014

19. Cettomal D, Kwasa J, Kendi C, Birbeck GL, Price RW, Bukusi EA, et al. Utility of quantitative sensory testing and screening tools in identifying hivassociated peripheral neuropathy in western kenya: pilot testing. PLoS One; 2010.5:e14256. DOI: 10.1371/journal.pone.0014256

20. Nebuchennykh M, Løseth S, Lindal S, Mellgren SI. The value of skin biopsy with recording of intraepidermal nerve fiber density and quantitative sensory testing in the assessment of small fiber involvement in patients with different causes of polyneuropathy. J Neurol; 2009.256:1067-75. DOI: 10.1007/s00415-009-5065-y

21. Simpson DM, Kitch D, Evans SR, et. al. HIV neuropathy natural history cohort study: assesment measures and risk factors. Neurology; 2006.66(11): 1679-87. DOI: 10.1212/01.wnl.0000218303.48113.5d

22. United States Department of Veteran Affairs. Peripheral Neuropathy and HIV - HIV/AIDS. http://www.hiv.va.gov/provider/manual-primarycare/peripheral-neuropathy-tooll.asp. 2010.

23. Ellis RJ, Evans SR, Clifford DB, et al. Clinical validation of the neuroscreen. $\mathrm{J}$ Neurovirol; 2005.11(6): 503-11. DOI: $10.1080 / 13550280500384966$

24. Muntean C, Cătălin B, Tudorică V, Moța M. Efficiency of michigan neuropathy screening instrument and nerve conduction studies for diagnosis of diabetic distal symmetric polyneuropathy. Rom J Diabetes Nutr Metab Dis; 2016.23(1):055065. DOI: 10.1515/rjdnmd-2016-0007

25. Bril V, Tomioka S, Buchanan RA, Perkins BA. Complications reliability and validity of the modified toronto clinical neuropathy score in diabetic sensorimotor polyneuropathy. journal compilation. diabetes uk. Diabetic Medicine; 2009.26:240-246. DOI: $10.1111 / \mathrm{j} .1464-5491.2009 .02667 . x$

26. Mete T, Aydin Y, Saka M, Yavuz HC, Bilen S, Yalcin $\mathrm{Y}$, et al. Comparison of efficiencies of michigan neuropathy screening instrument, neurothesiometer, and electromyography for diagnosis of diabetic neuropathy. International Journal of Endocrinology: 821745. 2013. DOI: 10.1155/2013/821745

27. Herman WH, Pop-Busui R, Braffet BH, Martin cl, cleary pa, albers jw et al. use of the michigan neuropathy screening instrument as a measure of distal symmetrical peripheral neuropathy in type 1 diabetes: results from the diabetes control an complications trial/epidemiology of diabetes interventions and complications. Diabet Med; 2012.29(7): page 937-944. DOI: 10.1111/j.14645491.2012.03644.x.

28. Moghtaderi A, Bakhshipour A, Rashidi H. Validation of michigan neuropathy screening instrument for diabetic peripheral neuropathy. Clinical Neurology and Neurosurgery 108; 2006.477-81. DOI: 10.1016/j.clineuro.2005.08.003

29. Craig BA., Strauss MB, Daniller A, Miller SS. Foot sensation testing in the patient with diabetes: introduction of the quick \& easy assessment tool. Wounds: 2013.26;(8)221-31. PubMed: https://www.ncbi.nlm.nih.gov/pubmed/25860638

30. Ponirakis G, Odriozola MN, Odriozola S, Petropoulos IN, Azmi IN, Fadavi H, et. al. NerveCheck: an inexpensive quantitative sensory testing device for patients with diabetic neuropathy. International Diabetes Federation. Diabetes Res Clin Pract; 2016. DOI: 10.1016/j.diabres.2015.12.023

31. Dubey TN, Raghuvanshi SS, Sharma H, Saxena R. HIV neuropathy in pre-haart patients and it's correlation with risk factors in central india. Neurology India; 2013.61(5):478-80. DOI: 10.4103/0028-3886.121912

32. Sithinamsuwan P, Punthanamongkol S, Valcour V, Onsanit S, Nidhinandana S, Thitivichianlert S, et. al. Frequency and characteristic of hiv-associated sensory neuropathy among hiv patients in bangkok, thailand. J Acquir Immune Defic Syndr; 2008.49(4):456-58. DOI: [10.1097/QAI.0b013e318186eb03]

33. Konchalard K, Wangphonpattanasiri K. Clinical and electrophysiologic evaluation of peripheral neuropathy in a group of hiv-infected patients in thailand. $\mathbf{J}$ Med Assoc Thai; 2007.90(4):774-80

34. Luma HN, Tchaleu BCN, Doualla MS, Temfack E, Sopouassi VNK, Mapoure YN, et al. Hiv-associated sensory neuropathy in hiv-1 infected patients at the douala general hospital in cameroon: a cross sectional study. AIDS Research and Theraphy; 2012.9:35. DOI: [10.1186/1742-6405-9-35]

35. Cettomai D, Kwasam JK, Birbeck GL, Price RW, Cohen CR, Bukusi EA., et al. Screening for hivassociated peripheral neuropathy in resource-limited setting. Muscle Nerve; 2013.48:516-24. DOI: [10.1002/mus.23795]

36. Nienov OH, Dias LS, Parisi MCR, Schmid H. Sensitivity and specificity of neuropathy diabetes score, neuropathy symptoms score, diabetic neuropathy score and esthesiometry compared with the gold standards michigan neuropathy screening instrument (mnsi) and beck depression inventory (BDI); 2015.7 (Suppl 1):A196. DOI: [10.1186/17585996-7-S1-A199]

37. Woldeamanuel YW, Kamerman PR, Veliotes DG, Phillips TJ, Asboe D, Boffito M, et. al. Development, validation, and field-testing of an instrument for clinical assessment of hiv-associated neuropathy and neuropathic pain in resource-restricted and large population study settings. clinical hiv-associated neuropathy tool (chant); 2016. DOI: 10.1371/journal.pone.0164994

38. Meijer JWG, Smit AJ, Lefrandt JD, Van Der Hoeven $\mathrm{JH}$, Hoogenberg K, Links TP. Back to basics in diagnosing diabetic polyneuropathy with the tuning fox. diabetes care; 2005.28:2201-5. PubMed: https://www.ncbi.nlm.nih.gov/pubmed/16123490

39. Govind BT, Jyoti KT, Prakash MS, Milind VB. Clinical and electrophysiologic evaluation of peripheral neuropathy in hiv infected patients. 
National Journal of Basic Medical Sciences; 2014. 4(4):227-33

40. Purbasari B, Anggraini VA, Pratiwi MD, Husna M, Kurniawan SN. Diagnostic test of toronto and modified toronto scoring, monofilament test, and vibrate sensation test using $128 \mathrm{hz}$ tuning fork for diabetic polyneuropathy. Malang Neurology Journal; 2018.4:25-34. DOI: 10.21776/ub.mnj.2018.004.01.5 